University of Wollongong

Research Online

Faculty of Engineering - Papers (Archive)

Faculty of Engineering and Information

Sciences

23-6-2006

\title{
Metastable behavior of vortex matter in the electronic transport processes of homogenous superconductors
}

\author{
X. B. Xu \\ Nanjing University, China \\ Y. Liu \\ Nanjing University, China \\ H. Fangohr \\ University of Southampton, UK \\ L. Zhang \\ Nanjing University, China \\ Shichao Ding \\ University of Wollongong, sding@uow.edu.au
}

See next page for additional authors

Follow this and additional works at: https://ro.uow.edu.au/engpapers

Part of the Engineering Commons

https://ro.uow.edu.au/engpapers/218

\section{Recommended Citation}

Xu, X. B.; Liu, Y.; Fangohr, H.; Zhang, L.; Ding, Shichao; Wang, Z. H.; Liu, S. L.; Wu, G. J.; and Shao, H. M.: Metastable behavior of vortex matter in the electronic transport processes of homogenous superconductors 2006.

https://ro.uow.edu.au/engpapers/218

Research Online is the open access institutional repository for the University of Wollongong. For further information contact the UOW Library: research-pubs@uow.edu.au 
Authors

X. B. Xu, Y. Liu, H. Fangohr, L. Zhang, Shichao Ding, Z. H. Wang, S. L. Liu, G. J. Wu, and H. M. Shao 


\title{
Metastable behavior of vortex matter in the electronic transport processes of homogenous superconductors
}

\author{
X. B. Xu, ${ }^{1, *}$ Y. Liu, ${ }^{1}$ H. Fangohr, ${ }^{2}$ L. Zhang, ${ }^{1}$ S. Y. Ding,,${ }^{1,3}$ Z. H. Wang, ${ }^{1}$ S. L. Liu, ${ }^{1}$ G. J. Wu,${ }^{1}$ and H. M. Shao ${ }^{1}$ \\ ${ }^{1}$ National Laboratory of Solid State Microstructures, Department of Physics, Nanjing University, \\ Nanjing 210093, People's Republic of China \\ ${ }^{2}$ Department of Electronics and Computer Science, University of Southampton, Southampton SO17 1BJ, United Kingdom \\ ${ }^{3}$ Institute for Superconducting and Electronic Materials, University of Wollongong, Wollongong, NSW 2522, Australia
}

(Received 19 February 2006; revised manuscript received 16 April 2006; published 23 June 2006)

\begin{abstract}
We study numerically the effect of vortex pinning on the hysteresis voltage-temperature $(V-T)$ loop of vortex matter. It is found that different types of the $V$ - $T$ loops result from different densities of vortex pinning center. An anticlockwise $V$ - $T$ loop is observed for the vortex system with dense pinning centers, whereas a clockwise $V$ - $T$ loop is brought about for vortices with dilute pinning centers. It is shown that the size of the $V$ - $T$ loop becomes smaller for lower experimental speed, higher magnetic field, or weak pinning strength. Our numerical observation is in good agreement with experiments.
\end{abstract}

DOI: 10.1103/PhysRevB.73.214521

PACS number(s): 74.25.Fy, 74.25.Qt

Continuous efforts have been made to understand the metastable phenomena in vortex matter for low- $T_{c}$ and high- $T_{c}$ superconductors. ${ }^{1-10}$ Generally, the metastable state is accessible when the experimental speed is higher than the response speed of the vortices to the external parameter such as temperature and magnetic field. For a vortex assemble, as the response speed of the vortex system changing from its initial state to its final one is too small to reach its final equilibrium state, vast metastable states that the system passes through are observed. As a result, relaxation which is the time evolution of the metastable state occurs. ${ }^{2}$ Then one can observe different states at different time windows. On the other hand, if the experimental speed is smaller by far than the response speed of a vortex system, one can observe in fact its final equilibrium state and thus no metastable state can be probed. This is the so-called "quasistatic state" process. Therefore, one can study the metastability of vortex matter which is characterized by current-voltage $I-V$ or $R-T$ loops in transport measurements by adjusting either the experimental speed (different scanning speed of temperature $d T / d t$, field $d H / d t$, or current $d I / d t)$ or the system response speed by changing $T, H, I$ or the vortex pinning in experiments for different systems, such as the untwinned ${ }^{4-6}$ or the twinned $\mathrm{YBa}_{2} \mathrm{Cu}_{3} \mathrm{O}_{7},{ }^{8}$ the pure ${ }^{9}$ or the doped $2 \mathrm{H}-\mathrm{NbSe}_{2},{ }^{10}$ as well as in simulation. ${ }^{11,12}$ Despite a large number of research which is mainly associated with the effects of external parameters on metastability, the role of vortex pinning is still not clear.

In this work, we use a standard two-dimensional Langevin simulation to study the effect of vortex pinning, temperature scanning speed (TSS), as well as magnetic field on the hysteresis $V$ - $T$ loop. We demonstrate how the competition between the experimental speed and the response speed of vortex systems with different densities of pinning centers affects the direction and size of the $R-T$ loop.

We model a transverse two-dimensional slice (in the $x-y$ plane) of an infinite superconducting slab containing rigid vortices that are parallel to the sample edges $(\mathbf{B}=B \hat{\mathbf{z}})$. The overdamped Langevin equation of motion for a vortex in position $\mathbf{r}_{i}$ is

$$
\mathbf{F}_{i}=\sum_{j \neq i}^{N_{\mathrm{v}}} \mathbf{F}^{\mathrm{vv}}\left(\mathbf{r}_{i}-\mathbf{r}_{j}\right)+\sum_{k}^{N_{\mathrm{p}}} \mathbf{F}^{\mathrm{vp}}\left(\mathbf{r}_{i}-\mathbf{r}_{k}^{\mathrm{p}}\right)+\mathbf{F}^{\mathrm{L}}+\mathbf{F}_{i}^{\mathrm{T}}=\eta \frac{d \mathbf{r}_{i}}{d t},
$$

where $\mathbf{F}_{i}$ is the total force acting on vortex $i$ due to vortexvortex interactions $\left(\mathbf{F}^{\mathrm{vv}}\right)$, pinning centers $\left(\mathbf{F}^{\mathrm{vp}}\right)$, the driving current $\mathbf{J}\left(\mathbf{F}^{\mathrm{L}} \propto \phi_{0} \mathbf{J} \times \hat{\mathbf{z}}\right)$ and thermal fluctuations $\left(\mathbf{F}^{\mathrm{T}}\right), \eta$ is the Bardeen-Stephen friction coefficient $\left(\eta \propto \phi_{0} B_{\mathrm{c} 2} / \rho_{\mathrm{n}}\right), N_{\mathrm{v}}$ is the number of vortices, $N_{\mathrm{p}}$ is the number of pinning centers, and $\mathbf{r}_{k}^{\mathrm{p}}$ is the position of the $k$ th pinning center. We chose $\mathbf{F}^{\mathrm{vv}}\left(\mathbf{r}_{i}-\mathbf{r}_{j}\right)=\left(\phi_{0}^{2} s\right)\left(2 \pi \mu_{0} \lambda^{2}\right)^{-1}\left(\mathbf{r}_{i}-\mathbf{r}_{j}\right)\left(\left|\mathbf{r}_{i}-\mathbf{r}_{j}\right|\right)^{-2}$, where $\phi_{0}$ is the flux quantum, $s$ is the length of the vortex, $\mu_{0}$ is the vacuum permeability, and $\lambda$ is the temperature dependent penetration depth. We employ periodic boundary conditions and cut off the logarithmic vortex-vortex repulsion potential smoothly. ${ }^{13}$ The random pinning center exerts an elementary pinning force on the vortices: $\mathbf{F}^{\mathrm{vp}}\left(\mathbf{r}_{i}-\mathbf{r}_{k}^{\mathrm{p}}\right)$ $=-f_{\mathrm{pv} 0} r_{i k} \exp \left[-\left(r_{i k} / r_{\mathrm{p}}\right)^{2}\right] \hat{\mathbf{r}}_{i k} / r_{\mathrm{p}}$, where $f_{\mathrm{pv} 0}$ tunes the strength of this force at zero temperature, $r_{\mathrm{p}}$ which was assumed to equal to $0.2 \lambda$ determines its range. The thermal force is implemented with a Box-Müller random number generator and has properties $\left\langle F_{i}^{\mathrm{T}}\right\rangle=0$ and $\left\langle F_{i}^{\mathrm{T}}(t) F_{j}^{\mathrm{T}}\left(t^{\prime}\right)\right\rangle$ $=2 \eta k_{\mathrm{B}} T \delta_{i j} \delta\left(t-t^{\prime}\right)$ at a given temperature $T$. To reflect the competing roles of $\mathbf{F}^{\mathrm{vv}}, \mathbf{F}^{\mathrm{vp}}$, and $\mathbf{F}^{\mathrm{T}}$ as a function of temperature, we use $\lambda(T) / \lambda(0)=\xi(T) / \xi(0)=\left(1-T / T_{\mathrm{c}}\right)^{-1 / 2}$. $^{1}$ The average velocities of vortices $\left\langle v_{x}\right\rangle=\frac{1}{N_{\mathrm{v}}} \sum_{i}^{N_{\mathrm{v}}} v_{i x}$ which is proportional to the mobility of the vortices and the resulting voltage (i.e., resistance $R$ ). We normalize lengths by $\lambda_{0}=\lambda(0)$, the forces by $f_{0}=\left(\phi_{0}^{2} s\right)\left(2 \pi \mu_{0} \lambda_{0}^{3}\right)^{-1}$ and time by $t_{0}=\lambda_{0} \eta(0) / f_{0}$. All quantities shown in the following figures are expressed in the simulation unit. This simulation is always performed on a cooling/heating cycle with a constant driving force $\left(F^{L}\right.$ $=0.5 f_{0}$ ) for the initial ordered vortex state. The random initial vortex arrangement has also been considered in this simulation, but the results obtained are qualitatively the same as those for the ordered initial vortex state (see below). Besides, our results are insensitive to the choice of the number of vortices being more than 400 .

Figure 1 illustrates a typical anticlockwise $V$ - $T$ loop and 


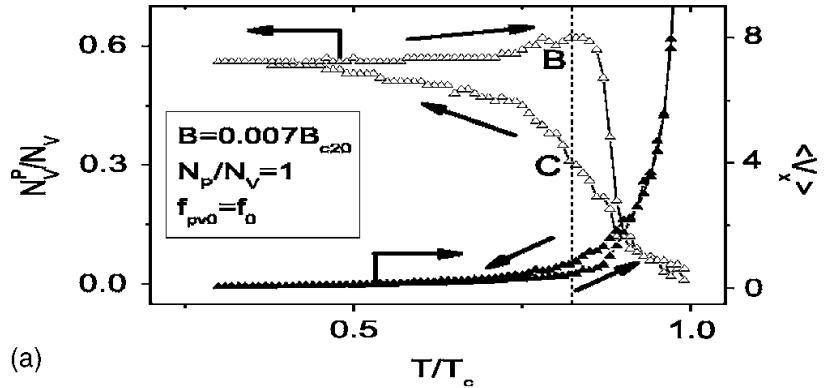

(b)

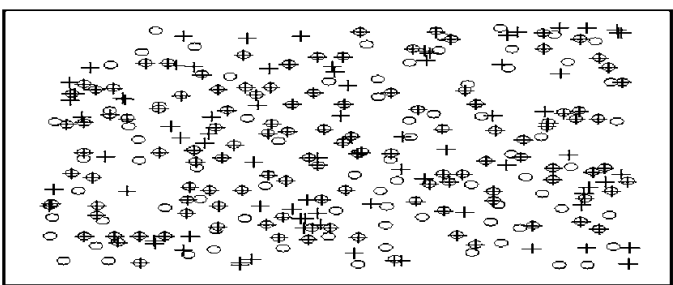

(c)

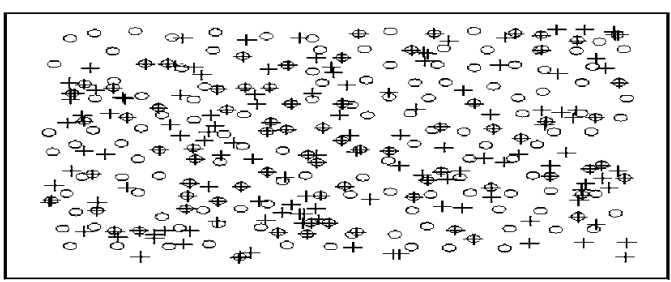

FIG. 1. (a) Typical anticlockwise $V-T$ loop (solid triangles) and the corresponding $N_{\mathrm{v}}^{\mathrm{p}} / N_{\mathrm{v}}-T$ loop (open triangles). $N_{\mathrm{v}}^{\mathrm{p}}$ is the number of pinned vortices. The temperature is varied in the direction indicated by the arrows. Solid lines are guide for eyes. (b) The vortices (open circles) and pinning centers (crosses) at position $B$ in (a). (c) The vortices (open circles) and pinning centers (crosses) at position $C$ in (a).

the corresponding number of pinned vortices $\left(N_{\mathrm{v}}^{\mathrm{p}}\right)$ normalized by $N_{v}$ versus the temperature curve $\left(N_{\mathrm{v}}^{\mathrm{p}} / N_{\mathrm{v}}-T\right)$ in one cooling/heating cycle for the vortex system with relative dense and weak pinning centers $\left(N_{p}=N_{v}, f_{p v 0}=f_{0}\right)$. With decreasing temperature, the number of pinned vortices increases at first because of the enhanced pinning forces for lower temperatures. However, the speed of the vortices moving into the random weak pinning potentials is very slow due to the small $f_{p v 0}$, which results in the slow but continuous increment of the number of pinned vortices $N_{\mathrm{v}}^{\mathrm{p}}$ till the temperature reaches $T_{B}$ on heating. At still higher temperatures, the pinned vortices decrease quickly because of the stronger thermal fluctuation. That is to say, the number of pinned vortices $N_{\mathrm{v}}^{\mathrm{p}}$ is increasing not only on cooling but also in most temperature regimes on heating [see Figs. 1(b) and 1(c), respectively]. Therefore, the $N_{\mathrm{v}}^{\mathrm{p}} / N_{\mathrm{v}}-T$ curve manifests itself as the clockwise loop and the corresponding anticlockwise $V$ $-T$ loop. ${ }^{4}$ Because the response speed of the vortices increases with increasing temperature, there is no loop in the region of the temperature near $T_{c}$ where thermal fluctuation is important. In contrast, at very low temperatures where both elasticity and pinning interactions are strong and thermal fluctuation is weak, the loop is too small to be observed.

Figure 2 shows the influence of TSS on the $V$ - $T$ loop. It is seen that the size of the loops becomes smaller with the decrement of TSS. The reason is that the vortex system has

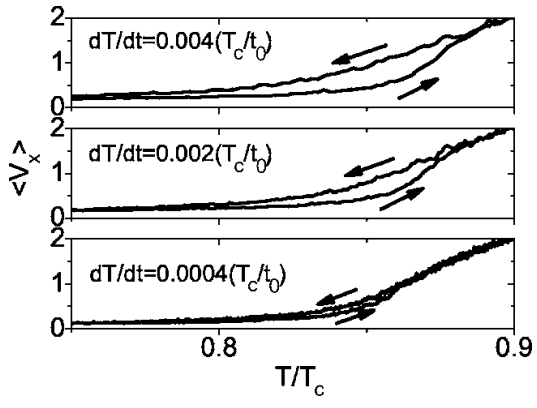

FIG. 2. Effect of the TSS on the $V-T$ loop. $B=0.007 B_{\mathrm{c} 20}, N_{p}$ $=N_{v}, f_{p v 0}=9 f_{0}$. TSS affects only the size of the $V-T$ loops but not their direction.

enough time to relax into more stable pinned states in a slower transport measurement. Therefore, for a very clean sample in which the vortex pinning is very weak and thus the response speed is very large, only has a fast experimental speed such as fast temperature or current scanning rates been used, the hysteresis loop can be observed. ${ }^{9}$

We have also investigated the effects of magnetic field on the hysteretic behaviors. Because the interaction between the vortices (the elasticity of vortex matter) increases with increasing field, the response speed of the vortex system will also increase correspondingly. Hence, it is expected that the $V$ - $T$ loop becomes smaller with increasing field. Shown in Fig. 3 is the influence of applied field on the $V$ - $T$ loop, which is just as our anticipation. The inset in Fig. 3 shows typical $V-T$ loops for different initial vortex states, indicating that the choice of initial vortex states does not influence qualitatively the results in this simulation. Therefore, we conclude that either TSS or field strength affects only the size and shape but not the direction of the loops.

Shown in Fig. 4 is a typical clockwise $V-T$ loop of the vortex system with a relative dilute and a strong pinning force $\left(N_{p}=0.1 N_{v}, f_{p v 0}=90 f_{0}\right)$. On cooling from the temperature near $T_{c}$, most of the vortices enter quickly into the random pinning potentials ( $N_{\mathrm{v}}^{\mathrm{p}}$ quickly increases) at temperatures near $T_{c}$ because of the strong elementary pinning force

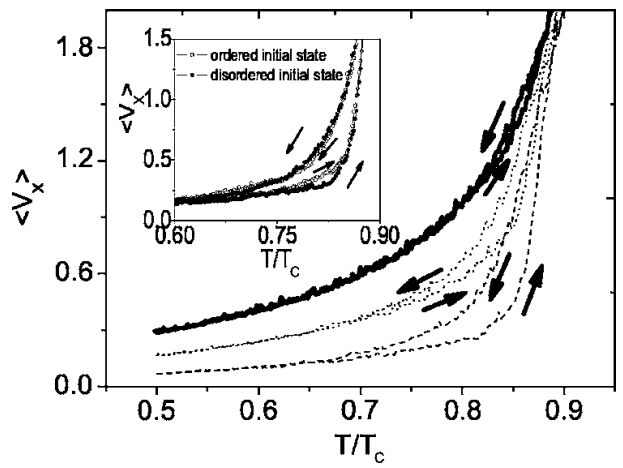

FIG. 3. Effect of the field on the $V-T$ loop. $d T / d t=0.04 T_{c} / t_{0}$, $f_{p v 0}=9 f_{0}, B=0.007 B_{\mathrm{c} 20}$ (dashed lines), $0.028 B_{\mathrm{c} 20}$ (dotted lines), and $0.056 B_{\mathrm{c} 20}$ (solid lines) corresponding to $N_{p}=N_{v}, 0.25 N_{v}$, and $0.125 N_{v}$. Inset: The $V-T$ loops for different initial vortex states. $d T / d t=0.04 T_{c} / t_{0}, f_{p v 0}=9 f_{0}, N_{p}=N_{v}$, and $B=0.007 B_{\mathrm{c} 20}$. The field affects only the size of the $V-T$ loops but not their direction. 


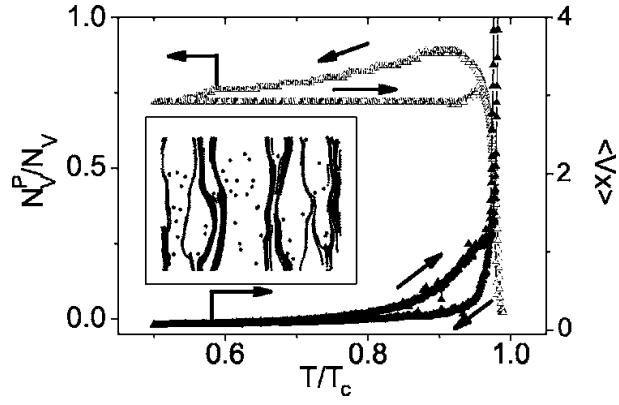

FIG. 4. Typical clockwise $V$-T loop (solid triangles) and the corresponding $N_{\mathrm{v}}^{\mathrm{p}} / N_{\mathrm{v}}-T$ curve (open triangles) for a systems with dilute pinning centers. $B=0.007 B_{\mathrm{c} 20}, \quad N_{p}=0.1 N_{v}, \quad d T / d t$ $=0.001 T_{c} / t_{0}$, and $f_{p v 0}=90 f_{0}$. Inset: Steady channel flow forms in the warming branch from $0.5 T_{c}$ to $0.91 T_{c}$. The circles and lines represent the random pinning centers and vortex trajectories, respectively.

and thermal fluctuation, which results in the occurrence of disordered pinned vortex states. With further decreasing temperature, vortex depinning occurs due to the faster increment of $F^{v v}\left(\sim \lambda^{-2}\right)$ than $F^{v p}\left(\sim \lambda^{-1}\right)$, as indicated by the corresponding decrement of the $N_{\mathrm{v}}^{\mathrm{p}} / N_{\mathrm{v}}$ in Fig. 4. The physics underlying the depinning is that a softer vortex is more efficient in vortex pinning than a stiffer one. Practically the measured voltage $V$ is determined by the global pinning force $F\left(\sim N_{\mathrm{v}}^{\mathrm{p}} F^{v p}\right)$. That is, $V$ is determined not only by the elementary pinning force $F^{v p}$ but also by the number of the pinned vortices $N_{\mathrm{v}}^{\mathrm{p}}$. If the contribution of $N_{\mathrm{v}}^{\mathrm{p}}$ is more important than the one of $F^{v p}, F\left(\sim J_{c} \mathrm{H}\right)$ decreases and thus $V$ increases. On the contrary, if the contribution of $F^{v p}$ is dominant, $F$ increases and, hence, $V$ decreases continuously, just as seen in Fig. 4. Furthermore, in the low temperature regime far from $T_{c}$, the depinned vortices form stable channels of vortex flow with the help of $F^{L}$ as well as $F^{T}$, which is shown in the inset of Fig. 4. ${ }^{12,14-19}$ The moving vortices in the channels are more in order than the pinned ones. This moving vortex structure maintains from $0.5 T_{c}$ to $0.91 T_{c}$ in the following heating process. At still higher temperatures on the heating branch, the moving structure is destroyed by strong thermal fluctuation. It is noted that at a given temperature $N_{\mathrm{v}}^{\mathrm{p}}$ of the vortex system with channel structure on the heating branch is smaller than the one on the cooling branch. Therefore, voltages are higher in the heating process than in the cooling one, i.e., the $V-T$ loop is clockwise. This suggests that the clockwise $V-T$ or $R-T$ hysteresis curves could be observed in those superconductors with few artificial strong pinning centers, just as observed in doped single crystals $2 \mathrm{H}-\mathrm{NbSe}_{2}$ (Ref. 10) and the twinned $\mathrm{YBa}_{2} \mathrm{Cu}_{3} \mathrm{O}_{7}{ }^{8}$

In order to check whether the strength or the density of the pinning centers can govern the direction of the $V$ - $T$ loop, we have to study the effect of the pinning strength $f_{p v 0}$ and density on the direction of the $V$ - $T$ loop. Displayed in Fig. 5 are the effects of pinning strength $f_{p v 0}$ on the $V-T$ loop. It is clear that the size of the $V-T$ loops becomes smaller with

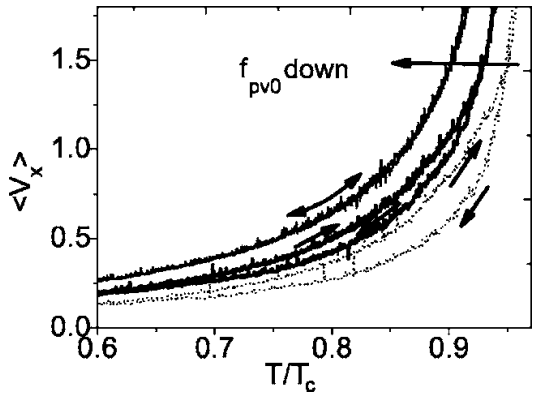

FIG. 5. Effect of pinning force strength $f_{p v 0}$ on the $V$-T loops. $B=0.007 B_{\mathrm{c} 20}, N_{p}=0.1 N_{v}, d T / d t=0.001 T_{c} / t_{0}, f_{p v 0}=$ (right) $60 f_{0}$, $45 f_{0}, 30 f_{0}$ (left). The size of the $V$ - $T$ loop becomes smaller with the decrement of $f_{p v 0}$, but its direction does not change.

decreasing $f_{p v 0}$, but the clockwise direction does not change. That is to say, whether the loop is clockwise or anticlockwise is not determined by the vortex pinning strength (also by TSS or field).

Figure 6 demonstrates the effects of the density of the pinning center on the $V$ - $T$ loop. One can see a crossover in the $V$ - $T$ curves, showing directly the change of the direction as well as the size of the loops for different density $N_{\mathrm{p}} / N_{\mathrm{v}}$. For a larger $N_{\mathrm{v}}^{\mathrm{p}} / N_{\mathrm{v}}$, such as $N_{\mathrm{p}} / N_{\mathrm{v}}=10$ (see curve $A$ in Fig. $6)$, no hysteresis can be seen, suggesting that it is difficult to probe the metastable states by the $V$ - $T$ loop measurement for a sample with the denser and weaker pinning centers. Whereas with decreasing $N_{\mathrm{p}} / N_{\mathrm{v}}$, it is noted that an anticlockwise $V$ - $T$ loop (curve $B$ in Fig. 6), a crossover one (curve $C$ in Fig. 6), and a clockwise one (curve $D$ in Fig. 6, the anticlockwise part disappears gradually) occur successively. This confirms the dominant rule of pinning center density in determining the direction of the $V-T$ loop. Besides, such a crossover of the $V$ - $T$ loop (curve $C$ in Fig. 6) has not been reported so far as we know.

In summary, we have performed simulations to study the effects of vortex pinning on metastable phenomenon in the transport process. It is shown that the vortex systems with

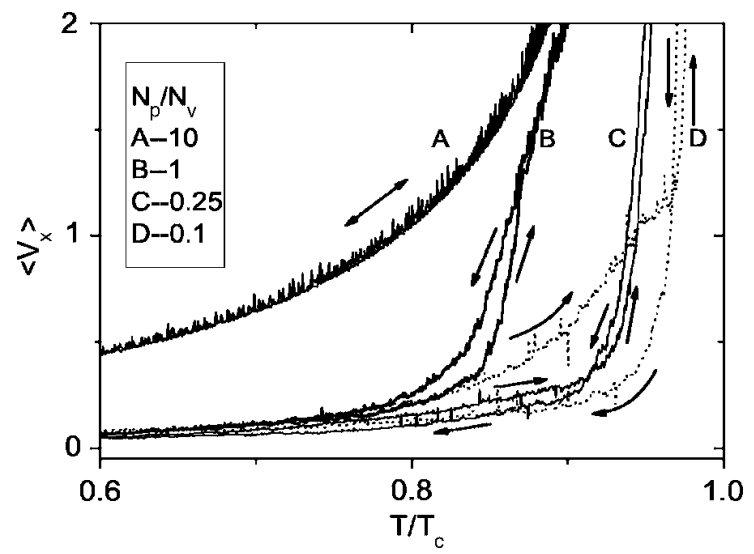

FIG. 6. Effects of the vortex pinning density on the $V$ - $T$ loops. $B=0.007 B_{\mathrm{c} 20}, d T / d t=0.001 T_{c} / t_{0}, N_{p} f_{p v 0} / N_{\mathrm{v}} \equiv 9 f_{0}$. The direction of the $V$ - $T$ loops depends on the vortex pinning density. 
very dense pinning centers will manifest their metastable states as an anticlockwise $V$ - $T$ loop. And the density of pinning center plays a crucial role in determining the direction of the $V-T$ loop. Meanwhile the pinning strength (also mag- netic field and TSS) can only influence the size of the $V$ - $T$ loop. The simulation results agree well with the reported experiments and predict the existence of crossover in the $V-T$ loops.
*Corresponding author. Email address: xxb@nju.edu.cn

${ }^{1}$ G. Blatter, M. V. Feigel'man, V. B. Geshkenbein, A. I. Larkin, and V. M. Vinokur, Rev. Mod. Phys. 66, 1125 (1994); E. H. Brandt, Rep. Prog. Phys. 58, 1465 (1995).

${ }^{2}$ Y. Yeshurun, A. P. Malozemoff, and A. Shaulov, Rev. Mod. Phys. 68, 911 (1996).

${ }^{3}$ T. Giamarchi and S. Bhattacharya, cond-mat/0111052 (unpublished).

${ }^{4}$ J. A. Fendrich, U. Welp, W. K. Kwok, A. E. Koshelev, G. W. Crabtree, and B. W. Veal, Phys. Rev. Lett. 77, 2073 (1996).

${ }^{5}$ H. Safar, P. L. Gammel, D. A. Huse, and D. J. Bishop, J. P. Rice, and D. M. Ginsberg, Phys. Rev. Lett. 69, 824 (1992).

${ }^{6}$ W. Jiang, N.-C. Yeh, D. S. Reed, U. Kriplani, and F. Holtzberg, Phys. Rev. Lett. 74, 1438 (1995).

${ }^{7}$ M. Charalambous, J. Chaussy, P. Lejay, and V. Vinokur, Phys. Rev. Lett. 71, 436 (1993).

${ }^{8}$ S. O. Valenzuela, B. Maiorov, E. Osquiguil, and V. Bekeris, Phys. Rev. B 65, 060504(R) (2002).

${ }^{9}$ Z. L. Xiao, E. Y. Andrei, and M. J. Higgins, Phys. Rev. Lett. 83, 1664 (1999); Z. L. Xiao, E. Y. Andrei, P. Shuk, and M. Greenblatt, ibid. 85, 3265 (2000); 86, 2431 (2001), and references therein.
${ }^{10}$ W. Henderson, E. Y. Andrei, M. J. Higgins, and S. Bhattacharya, Phys. Rev. Lett. 77, 2077 (1996).

${ }^{11}$ P. Moretti, M. Carmen Miguel, and S. Zapperi, Phys. Rev. B 72, 014505 (2005).

${ }^{12}$ C. J. Olson, C. Reichhardt, R. T. Scalettar, G. T. Zimányi, and N. Grønbech-Jensen, Phys. Rev. B 67, 184523 (2003); C. J. Olson, C. Reichhardt, and F. Nori, Phys. Rev. Lett. 81, 3757 (1998).

${ }^{13}$ H. Fangohr, A. Price, S. Cox, P. A. J. de Groot, G. J. Daniell, and K. S. Thomas, J. Comput. Phys. 162, 372 (2000).

${ }^{14}$ S. Ryu, M. Hellerqvist, S. Doniach, A. Kapitulnik, and D. Stroud, Phys. Rev. Lett. 77, 5114 (1996).

${ }^{15}$ C. Reichhardt, G. T. Zimányi, and N. Grønbech-Jensen, Phys. Rev. B 64, 014501 (2001).

${ }^{16}$ H. Fangohr, S. J. Cox, and P. A. J. de Groot, Phys. Rev. B 64, 064505 (2001).

${ }^{17}$ A. B. Kolton, D. Domínguez, and N. Grønbech-Jensen, Phys. Rev. Lett. 83, 3061 (1999).

${ }^{18}$ F. Pardo, F. de la Cruz, P. L. Gammel, E. Bucher, and D. J. Bishop, Nature (London) 396, 348 (1998).

${ }^{19}$ K. Moon, R. T. Scalettar, and G. T. Zimányi, Phys. Rev. Lett. 77, 2778 (1996). 